Bayero Journal of Pure and Applied Sciences: 10(1): 252 - 257

ISSN 2006 - 6996

\title{
DISTANCE TRACKING SCHEME FOR SEAMLESS HANDOVER IN IMS-BASED SYSTEMS WITH UMTS ACCESS NETWORK
}

\author{
${ }^{1}$ Adamu Aminu and ${ }^{2}$ Muhammad Lawal \\ Umaru Musa Yar'adua University, Katsina. P.M.B 2218, Katsina State, Nigeria \\ Mathematics and Computer Science Department \\ aminu@mail.ru: ${ }^{1}+2348031581658 ;{ }^{2}$ mlawalsafana@gmail.com: ${ }^{2}+2348034711530$
}

\section{ABSTRACT}

This paper proposes a fast and seamless handover scheme for systems based on IP Multimedia Subsystem (IMS) architectural framework with Universal Mobile Telecommunications System (UMTS) access network. In the scheme the location, direction and movement pattern of a Mobile Node $(M N)$ in a network cell are proactively tracked in order to determine the exact moment to trigger the pre-handover process with one of the adjacent cells considering MN's direction before the execution of Layer 2 handover. The performance of the proposed scheme was evaluated by comparing it with the traditional handover scheme and the evaluation shows that the traditional handover scheme introduced a handover delay of about $675.5 \mathrm{~ms}$, whereas the proposed scheme introduced handover delay of about $96.25 \mathrm{~ms}$, which is less than $100 \mathrm{~ms}$, hence the handover delay introduced by the proposed scheme fall within the specified range of best class service for voice traffic and streaming media over IP networks. Keywords: IMS, Handover delay, Distance Tracking, pre-handover, UMTS.

\section{INTRODUCTION}

Handover is a key process in mobile networks for mobility management and is defined as transferring of the mobile node's connection from one network coverage area to another while maintaining good quality of service (QoS) for the ongoing session (Banerjee et al, 2004; Kera, et al, 2015). Handover is a critical process and if performed incorrectly or not as fast as possible can result in dropping the ongoing session. Dropped sessions are particularly annoying to users, and the increased rate of dropped connections leads to the degraded QoS hence user dissatisfaction. Handover delay of more than 200 ms makes voice communication very unpleasant (Banerjee et al, 2004). Unfortunately normal handover process in IMS-based systems with UMTS access network tends to be very long and is intolerable to delay sensitive traffics (Banerjee et al, 2004; Kim, Joo and Kyoung-Hee, 2009). Therefore there is a need to devise an effective scheme to minimize the handover delay in IMS-based systems with UMTS access network which if implemented will facilitate fast and seamless handover (Banerjee et al, 2004; Kim, Joo and Kyoung-Hee, 2009).

In this paper we adopted the empirical propagation path loss model proposed in (Abhayawardhana et al, 2005; Han and Hwang, 2008) and incorporated it with pre-handover process which consists of the execution of preregistration procedures followed by SIP preINVITE procedure to markedly minimize handover delay in IMS-based systems with UMTS access network. The basic idea is to determine the location of the $M N$ within a network cell and the speed with which the $M N$ is moving in order to determine the right moment to commence the pre-registration procedures (acquiring new IP address, establishment of security association with the target (new) network and so on) and SIP Pre-INVITE procedure to prematurely reestablish the session before the complete detachment of $M N$ from old network i.e. before Layer 2 handover. The rest of this paper is organized as follows. Section 2 provides the description of the underline network architecture of the considered system and explains in detail the traditional handover process in IMS-based systems with UMTS access network. Section 3 describes the proposed distance tracking scheme and in section 4 the performance of the proposed scheme was evaluated. Finally section 5 concludes the paper.

UNDERLINE NETWORK ARCHITECTURE

In this paper UMTS release 5 is considered for demonstration purposes even though the proposed scheme can be applied with some modifications for later generation access networks. UMTS release 5 provides access to Internet services with the help of architectural framework known as IP Multimedia Subsystem (IMS) (Orhan et al, 2016; Camarillo and GarciaMartin, 2006; Kera, et al, 2015). The role of all the functional entities of the underline network architecture above can be found in (Orhan et al, 2016; Abdulbas et et al, 2012; Banerjee et al, 2004). 
Assume a MN connected to Home Network (old network) moves toward new network coverage area while on session with the correspondent node $(\mathrm{CN})$. MN's services must be handed over to the new network coverage area to ensure session continuity (Figure 1).

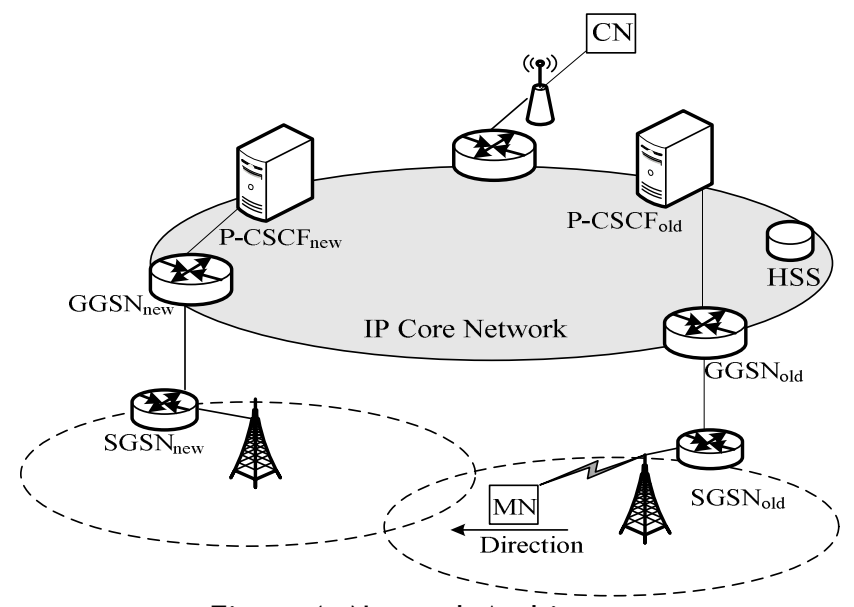

Figure 1: Network Architecture

To connect with a new network, MN must execute three procedures which are: GPRS attach procedure, Packet Data Protocol (PDP) context activation procedure and session reestablishment procedure, the first two procedures are collectively called data connection procedures, further detail on these procedures can be found in (Orhan et al, 2016; Banerjee et al, 2004). All these three procedures must be performed after Layer 2 handover (Link Switching Operation) and are done to ensure session continuity (Orhan et al, 2016; Abdulbaset et al, 2012; Banerjee et al, 2004).

We referred the above explained handover process as Traditional Handover Process in IMSbased systems with UMTS access network.

Distance Tracking SCHEME for Fast and Seamless HANDOVER IN IMS-BASED SYSTEMS WITH UMTS ACCESS NETWORK
In the Proposed handover scheme data connection procedures is prematurely executed with only one of the neighboring cells with higher probability of receiving the handover considering the movement direction of $M N$ then followed by SIP pre-INVITE procedure to prematurely reestablish a session with the $\mathrm{CN}$ (Note that the pre-handover process is to be executed before the complete detachment of the $\mathrm{MN}$ from the old network). To this end, each cell is divided into 2 zones using concentric circles based on the strength of the radio signal received from the base station (BS) at the center of the cell. The inner zone is referred to as the cell-core. The outer zone is then further subdivided into six (6) sectors using cell sectoring technique (Han, Lee, \& Hwang, 2008). We referred these individual sectors to pre-handover regions. This process is illustrated in Figure 2.

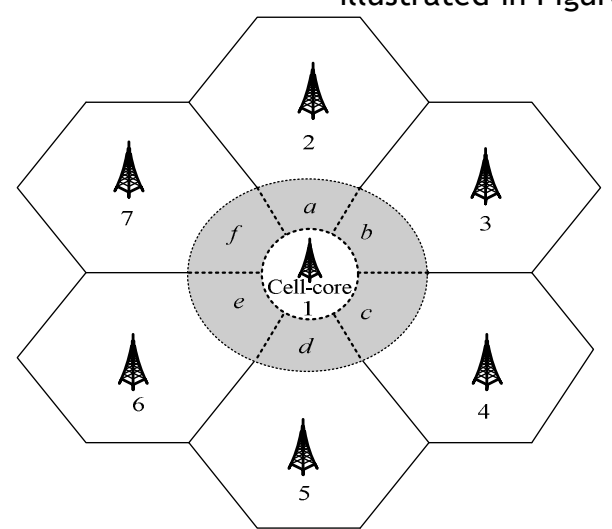

Figure 2: Cell Division

If the $M N$ is in pre-handover region $a$ (sector $a$ ), handover is performed only with cell 2 . When a it can only move to sector $b$, sector $f$ or cell 2 . If the $M N$ moves to sector $b$ or $f$, then an intracell handover will take place which does not $M N$ is inside the cell-core, the probability of a handover request is considered to be low; as such pre-handover process will not be initiated. 
To decided when to execute pre-handover procedures it is important to know the location of the $M N$ within the current cell, to determine the location of $M N$ two variables were used: which are distance between $M N$ and base station denoted by $d$ and the radius of cell-core denoted by $r$. If $d>r$, then $M N$ is outside the cell-core else it is in the cell-core. $M N$ periodically measured its distance from the base station by executing Equation (1) (Abhayawardhana et al, 2005).

$$
d=10^{x} \text {, }
$$

Where $x=\frac{P L-46.30-33.90 \log f+13.82 \log h_{B S}+\varepsilon_{i}-\delta_{i}}{44.90-6.55 \log h_{B S}}$.

Denoted by $f$ the frequency in $M H z, h_{B S}$ is the BS antenna height above ground level in meters (from $30 \mathrm{~m}$ to $200 \mathrm{~m}$ ); $d$ is the distance between the BS and the MN antenna in

kilometers. The index $i$ denote the environment (urban, suburban or rural). The parameter $\delta_{i}$ is the correction factor for the environment and is:

$$
\delta_{i}=\left\{\begin{array}{ll}
0 \mathrm{~dB} & \text { if } i=\text { Rural }=\text { Suburban } \\
3 \mathrm{~dB} & \text { if } i=\text { Urban }
\end{array},\right.
$$

The parameter $\varepsilon_{i}$ is a correction factor for effective mobile antenna height, which is a function of the coverage area and is defined as:

$$
\varepsilon_{i}=\left\{\begin{array}{l}
(1.10 \log f-0.70) D-1.56 \log f+0.80 \text { if } i=\text { Rural }=\text { Suburban } \\
3.20 \log 138.06 D^{2}-4.97 \text { if } i=\text { Urban }
\end{array}\right.
$$

Where $D$ is the MN's distance above the ground level.

Since, the MNs have difference velocities, we made the size of cell-core to be the function of the MN's velocity so that the higher the velocity the smaller the cell-core and the lower the velocity the larger the cell-core this process is illustrated in Figure 3.

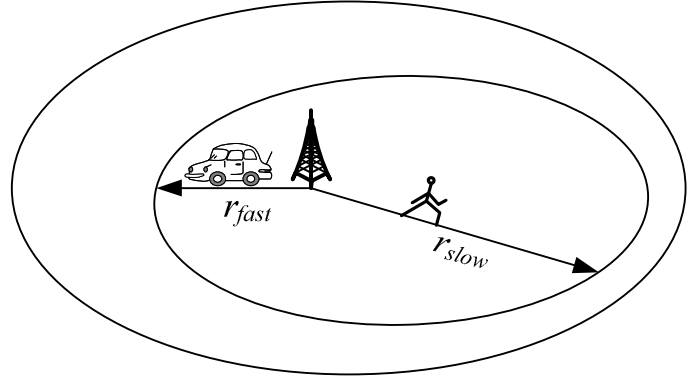

Figure 3: The change of $r$ with a change in $V$

The radius $r$ is computed using Equation (4).

$$
r(V)=R-V \cdot T \text {. }
$$

Where $R$ is the radius of the entire cell and $V$ is the MN's velocity and is defined as:

$$
V=\frac{d\left(t_{i}\right)-d\left(t_{i-1}\right)}{t_{i}-t_{i-1}}
$$

Where $t_{i}$ the time at which the distance is measured at $i^{\text {th }}$ epoch and that distance is represented as $d\left(t_{i}\right)$.

Denoted by $T$ the average time required to execute data connection procedures and is defined as:

$$
T=T_{1}+T_{2}
$$

Where $T_{1}$ is the average time to execute data connection procedures and $T_{2}$ is the average time to execute pre-INVITE procedure.

MN keeps measuring distance $d$ by executing equation (1) and comparing the measured distance $d$ with $r$. If $d>r$, then the MN is assumed to be in pre-handover region, and it is at that moment the pre-handover process should start. First step of the pre-handover process is GPRS attach procedure with $\mathrm{SGSN}_{\text {new }}$ (SGSN of the target network). Then the PDP context activation procedure, where the $M N$ will obtain a new IP address and the IP address of P-CSCF new (P-CSCF of the target network). After successful completion of the above procedures, MN will 
send pre-INVITE request message to the $P$ $\mathrm{CSCF}_{\text {old }}(\mathrm{P}-\mathrm{CSCF}$ of the home network) querying it to reestablish a session using the initial session ID and the newly configured IP address on its behalf (while at the same time the task of switching from old network interface to that of new network is ongoing). P-CSCF old will send pre-INVITE message to $\mathrm{CN}$ through $\mathrm{P}-\mathrm{CSCF}_{\text {new }}$. After successful completion of the pre-INVITE procedure all packets sent by $\mathrm{CN}$ to the $\mathrm{MN}$ at this stage would be stored in the buffer of the $\mathrm{GGSN}_{\text {new }}$ (Kim, Joo \& Kyoung-Hee, 2009). After successful L2 handover (Link switching handover), the $M N$ will notify the $P-C_{S C F}$ new that it is fully attached to the new network and can receive all the packets sent from the $\mathrm{CN}$, $M N$ does that by sending handover completed message $(\mathrm{H} . \mathrm{O}$ completed $)$ to the $\mathrm{P}-\mathrm{CSCF}_{\text {new }}$. When $\mathrm{H} . \mathrm{O}$ completed message is received by $\mathrm{P}$ $\mathrm{CSCF}_{\text {new, }}$ then it will forward it to the GGSN $\mathrm{Gew}_{\text {n }}$ querying it to deliver the buffered packets from $\mathrm{CN}$ and to release the connection directly to the MN. The explained process is shown in Figure 4.

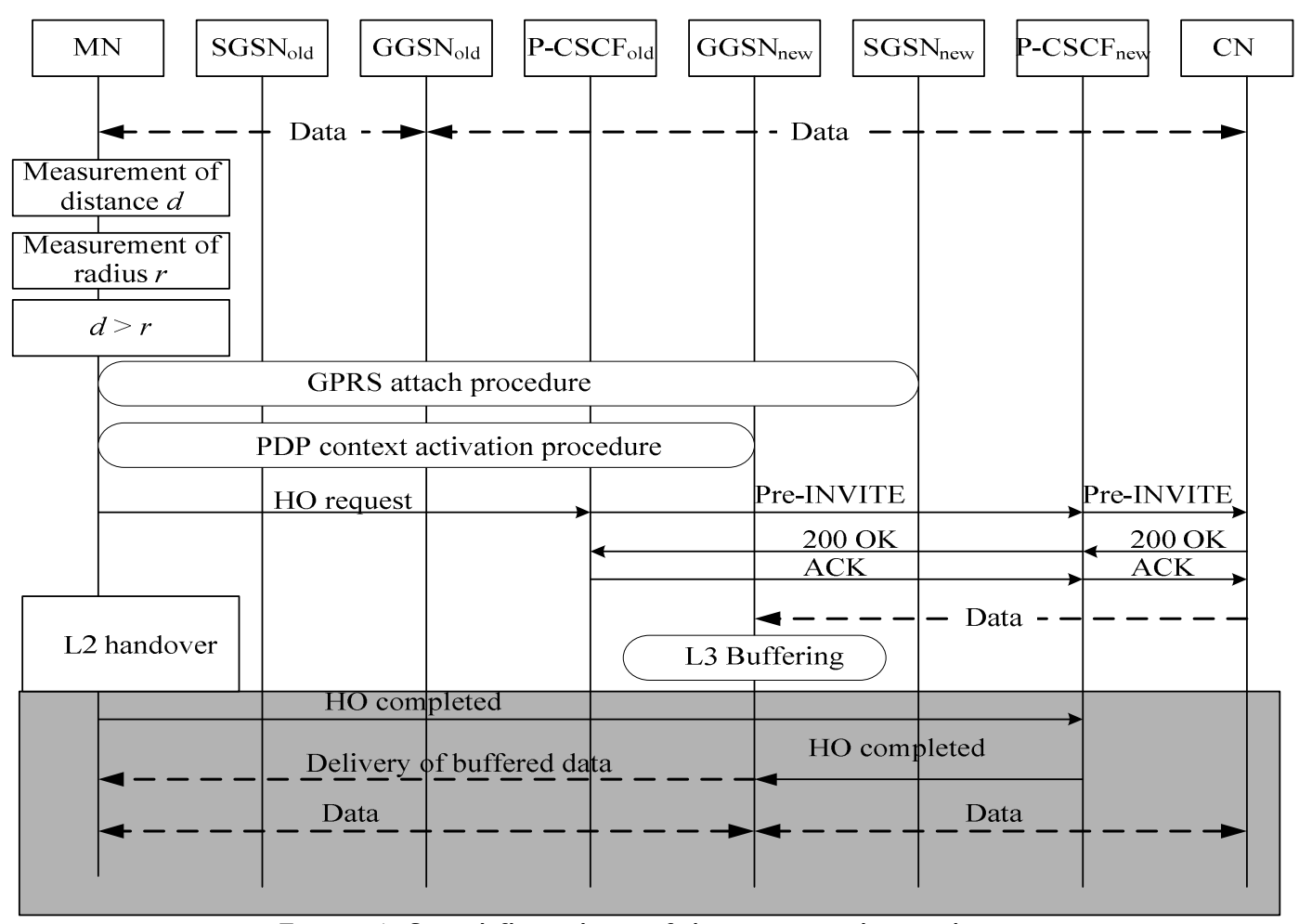

Figure 4: Signal flow chart of distance tracking scheme

\section{Performance Evaluation}

A simulation experiment was conducted to evaluate the effectiveness of the proposed scheme and it was implemented using MatLab (version 9.2, R2017a) software simulator. For that purpose all the three handover procedures mentioned in section 2 combined in a single diagram as shown in Figure 5. 


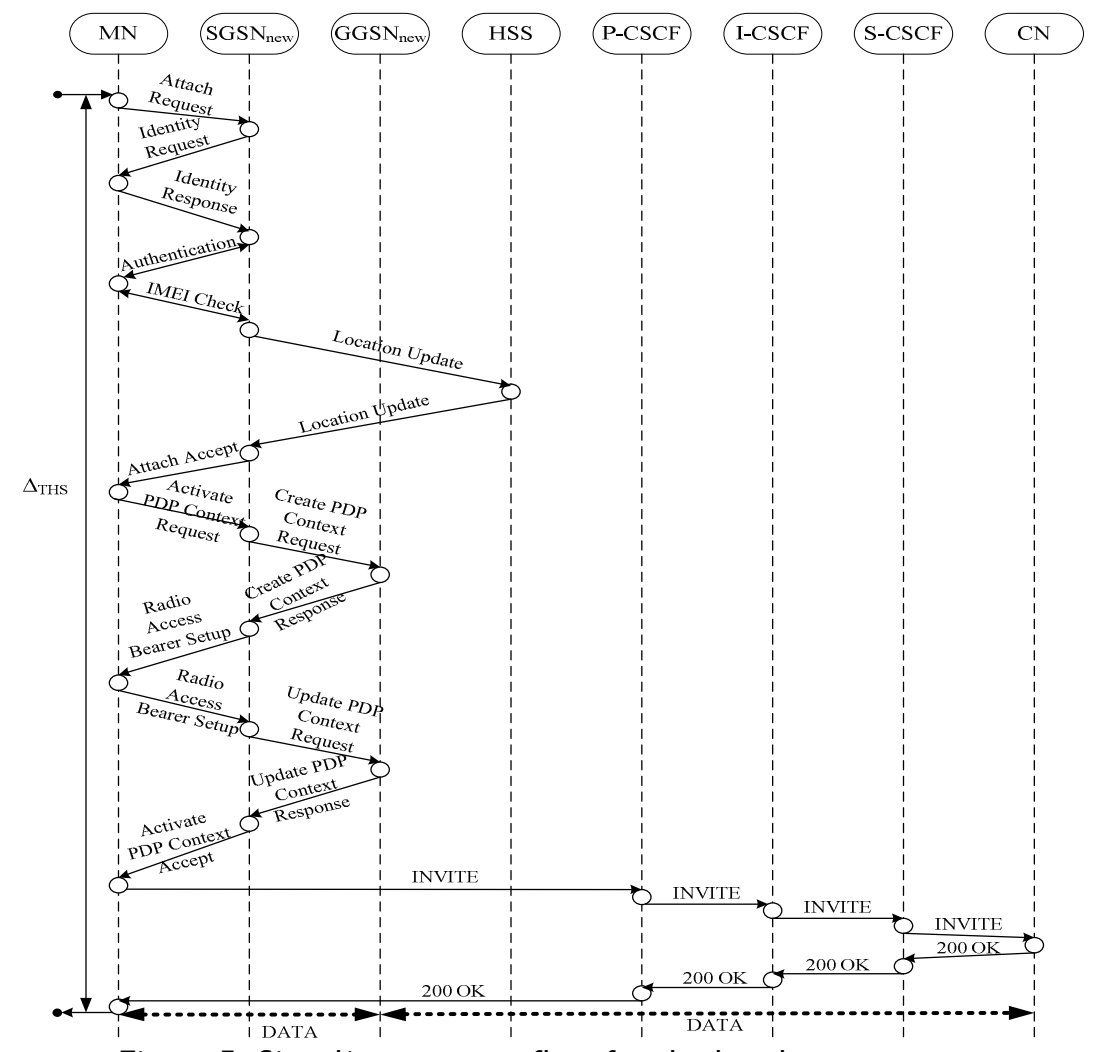

Figure 5: Signaling message flow for the handover process

The set $N=\{1,2,3,4,5,6,7,8\}$ was obtained by numbering the functional entities that are involved in the handover process from Figure 5. It was assumed that all external arrivals of requests to be a Poisson process with rate $\lambda$. The processing rate $\mu_{i}, i=1, \cdots, 8$ at each node is fixed. Signaling message flow between nodes of the network is described by routing matrix

$\boldsymbol{\Theta}=\left(\theta_{i j}\right)$, for $i, j \in N$.

The set $M$ contains all the messages required to be processed to accomplish the entire handover process, where $n_{m}$ the number of $m$ message's transitions before reaching its final destination node and $L_{m}$ the size of $m$-message in bits, $m \in M$.

The 1 -node and 8 -node were modeled as $M / M /$ inf type, whereas the rest of the nodes (2-, 3-, 4-, 5-, 6- and 7- nodes) were modeled as $M / M / 1 /$ inf type with service discipline FCFS. Handover process consists of three procedures as mentioned in section 2: GPRS attach procedure, PDP context activation procedure and the session reestablishment procedure using SIP re-INVITE message. The set $N$ was divided into three sets:

$$
\begin{aligned}
& N^{(1)}=\{1,2,4\}, \quad N^{(2)}=\{1,2,3\}, \\
& N^{(3)}=\{1,5,6,7,8\} .
\end{aligned}
$$

The first set $N^{(1)}$ contain the nodes that are involved in GPRS attach procedure, second set $N^{(2)}$ contain the nodes that are involved in PDP context activation procedure and the last set $N^{(3)}$ contain the nodes that are involved in session reestablishment procedure. The handover delay for the traditional handover scheme is denoted by $\Delta_{\text {THS }}$ and for the proposed scheme the handover delay is denoted by $\Delta_{\text {DTS }}$.

In the simulation conducted 50 cells were used with a honeycomb-like organization where the transmission ranges were modeled as circles enclosing the cells, each cell contained all the necessary nodes for MNs communications. Random waypoint mobility model was used where the MNs moved along a straight line from two destination points (waypoints) (Santi, 2012), 50 MNs were introduced and their velocities were defined by a uniform distribution.

For the experiment, it was assumed that the average processing rate at 1 -node and 8 -node is $0.4 \mathrm{~ms}$, average processing rate at 2-node, 3node and 4-node is $0.5 \mathrm{~ms}$ and the average processing rate at 5 -node, 6 -node and 7 -node is $0.4 \mathrm{~ms}$ (Banerjee et al, 2004). 
It was assumed also that the average size of GPRS attach messages is 43 bytes, the average size of PDP Context Activation messages is 573 bytes and average size of SIP messages for session reestablishment is 731 bytes (Ohta, 2007), the $M N$ speed was chosen between the interval of $1 \mathrm{~m} / \mathrm{s}$ to $20 \mathrm{~m} / \mathrm{s}$ in order to reflect the velocity of walking MNs and the velocity of fast moving MNs. Channel with $128 \mathrm{kbps}$ was considered.

Results obtained were presented in Figure 6, it can be observed from the graph that the

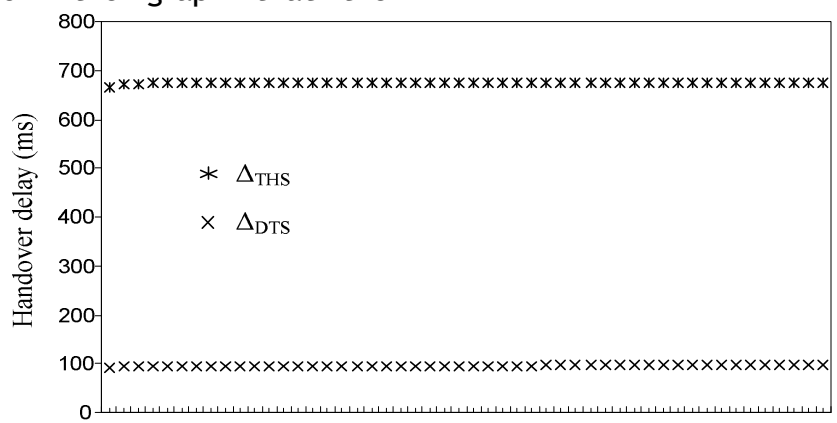

Figure 6: Handover delay for traditional handover scheme and the distance tracking scheme CONCLUSION

In this paper a fast and seamless handover scheme was proposed for IMS-based systems with UMTS access network. In the scheme the location, direction and movement pattern of a Mobile Node $(\mathrm{MN})$ in a network cell dictates the right moment to trigger the pre-handover process with one of the adjacent cells having the highest chance of receiving the handover. In the proposed scheme however, SIP protocol was slightly modified where pre-INVITE message was introduced to prematurely reestablish the

\section{REFERENCES}

Abdulbaset, S. et al. (2012). Session Initiation Protocol (SIP): An Evolutionary Study. Journal of communications, 7, (2), 89-105.

Abhayawardhana, V.S., Wassell, I.J., Crosby, D., Sellars, M.P. and Brown, M.J. (2005). Comparative Analysis of Empirical Propagation Path Loss Model for Fixed Wireless Access Networks. $61^{\text {st }}$ IEEE Vehicular Technology Conference, Stockholm, 73-77.

Banerjee, N. et al. (2004). Analysis of SIP-based mobility management in $4 \mathrm{G}$ wireless networks. Computer Communications, 27, 697-707.

Camarillo, G. and Garcia-Martin, M.A. (2006). The 3G IP Multimedia Subsystem (IMS): Merging the Internet and the Cellular Worlds, $2^{\text {nd }}$ edition. John Wiley \& Sons Ltd, ISBN: 0-470-01818-6.

Han, S., Lee, K. and Hwang, C. (2008). Region-based Shadow Registration Scheme for Efficient Mobility Management in the SIP. Journal of Information Science and Engineering, 24, 709725. average handover delay for the traditional handover scheme was around $675.5 \mathrm{~ms}$ for the MNs, which is significantly long, and such a delay may not be tolerated by the time sensitive services, however, the average handover delay for the proposed scheme was around $96.25 \mathrm{~ms}$ for the MNs, which fall within the specified range of best class service for voice traffic and streaming media over IP networks (Banerjee et al, 2004). session with the $\mathrm{CN}$ before Layer 2 handover. The performance of the proposed scheme was evaluated by comparing it with the traditional handover scheme and the evaluation shows that the traditional handover scheme introduced a handover delay of about $675.5 \mathrm{~ms}$, whereas the proposed scheme introduced handover delay of about $96.25 \mathrm{~ms}$, which is less than $100 \mathrm{~ms}$, hence the handover delay introduced by the proposed scheme fall within the specified range of best class service for voice traffic and streaming media over IP networks.

Kera, S. et al. (2015). A Review on Research Aspects of Vertical Handover Decision Based Intelligent Algorithm. International Journal of Innovative Research in Science, engineering and technology, 4, (8), 7207-7213.

Kim, S., Joo, S.K and Kyoung-Hee, S. (2009). Network-based Fast Handover for IMS Applications and Services. The 11th international conference on advanced communication technology. IEEE Communication Society, 1133-1136.

Ohta. (2007). Overload Control in a SIP Signaling Network. International Journal of Computer, Electrical, Automation, Control and Information Engineering, 1, (12), 4076-4081.

Orhan, O. et al. (2016). IP Multimedia Subsystem and its Feature Perspectives. International Journal of Computing Academic Research, 5, (4), 187194.

Santi, P. (2012). Mobility Models for Next Generation Wireless Networks: Ad Hoc, Vehicular and Mesh Networks, First Edition. Wiley and Sons, Ltd. 